\title{
THE MOST STABLE STRUCTURE FOR HARD SPHERES
}

\author{
by \\ Hans Koch ${ }^{1}$, Charles Radin * and Lorenzo Sadun ** \\ Department of Mathematics, University of Texas, Austin, TX
}

\begin{abstract}
The hard sphere model is known to show a liquid-solid phase transition, with the solid expected to be either face centered cubic or hexagonal close packed. The differences in free energy of the two structures is very small and various attempts have been made to determine which structure is the more stable. We contrast the different approaches and extend one.
\end{abstract}

\footnotetext{
1 Research supported in part by NSF Grant DMS-0322962

* Research supported in part by NSF Grant DMS-0354994

** Research supported in part by NSF Grant DMS-0401655
} 


\section{Introduction.}

Computer simulations of the hard sphere model in the late 1950's (see [B] for a review) showed a liquid/solid phase transition at packing fractions in the range 0.49 to 0.54 . Ever since that time there has been significant interest in determining the internal structure of that solid phase, since the model is one of the simplest possible showing both liquid and solid phases: the only interaction is that the spheres may not overlap. At least at high density, the solid is expected to have one of the structures associated with densest packing (packing fraction $\pi / \sqrt{18} \approx 0.74$ ), in particular the face centered cubic (fcc) and hexagonal close packed (hcp) structures, which commonly appear in materials. In 1968 Stillinger and coworkers [RSYS] used series expansions to try to answer the much simpler question of whether fcc or hcp is more stable. They computed the first 3 terms in a series for the entropy per sphere for both fcc and hcp and concluded that hcp had higher entropy per sphere and was therefore more stable; no error bars were given. We will extend this analysis to 2 more terms. Others used molecular dynamics or Monte Carlo simulations [HR,FL] to compare the fcc and hcp entropies, with inconclusive results. In 1997, using more advanced computer technology, Woodcock [W1,W2] did a molecular dynamics simulation and concluded that fcc is more stable, with error bars to back up this conclusion. The error bars and the magnitude of the entropy difference were called into question, but further simulations [BFMH,BWA,MH] agreed with Woodcock's qualitative claim that fcc is more stable than hcp. Finally, a geometric approach was recently applied to the first nontrivial term in Stillinger's expansion [RS]. This paper concerns the effort to extend the computation of the Stillinger expansion so as to determine the geometric mechanism behind stability.

\section{Analysis}

We consider the hard sphere model of classical statistical mechanics in the canonical ensemble, consisting of the uniform distribution of configurations of nonoverlapping spheres at fixed packing fraction. (The momentum variables can be integrated out, as they are decoupled from physical space variables in this model.) We assume, based on old simulations [B], that at high density (packing fraction $d \approx \pi / \sqrt{18} \approx 0.74$ ) the model is in a solid phase which is, moreover, either an fcc or hcp crystal. More specifically, the assumption is that the distribution gives overwhelming weight to configurations of spheres which are perturbations of either a perfect fcc or hcp structure at the appropriate density. We begin with an analysis of the meaning of "perturbation" and of error estimates for this problem.

One way to make definite the meaning of perturbations of the perfect fcc structure for $d \approx \pi / \sqrt{18}$ is as follows. Start with a finite block of spheres frozen in a perfect fcc arrangement at density below close packing (obtained by relative choice of sphere radius and lattice spacing), and with a subset $Q$ of $|Q|=N$ lattice sites for which we free up the associated spheres. As in [RSYS] we note that, as $d \rightarrow \pi / \sqrt{18}$, the restrictions in position of any sphere due to its neighbors can be approximated by neglecting the curvature of the spheres, obtaining linear restric- 
tions. Specifically, suppose our lattice spacing is $1, L_{j}$ represents the coordinates of the $j^{t h}$ lattice site, and $u_{j}$ represents the relative coordinates of the center of the sphere of diameter $1-\epsilon$ associated with this site. For nearest neighbor sites $j$ and $k$, the hard core restriction is $\left\|L_{j}+u_{j}-L_{k}-u_{k}\right\| \geq 1-\epsilon$ and the linearization is $\left(L_{j}+u_{j}-L_{k}-u_{k}\right) \cdot\left(L_{j}-L_{k}\right) \geq 1-\epsilon$, or $\left(u_{j}-u_{k}\right) \cdot\left(L_{j}-L_{k}\right) \geq-\epsilon$. So the entropy density $s$ of this class of configurations at high density is precisely $\ln (V) / N$, where $V$ is the volume of the convex polyhedron in $\mathbb{R}^{3 N}$ whose faces are the linear constraints on the positions of the $N$ moveable spheres due to the frozen spheres and each other.

Now that we have a class of configurations associated with each of fcc and hcp at high density, we consider the corresponding entropy densities $s_{f c c}$ and $s_{h c p}$. As shown in [RSYS], $s_{j}(d)$ diverges as $d \rightarrow \pi / \sqrt{18}$ and is of the form $\ln (\Delta d)+C_{j}+$ $O(\Delta d)$, where $\Delta d=\pi / \sqrt{18}-d$ and the $C_{j}$ are constants. In order to determine whether fcc or hcp is more stable one must estimate the difference $\Delta C=C_{h c p}-C_{f c c}$. All works we are considering claim that $|\Delta C| \approx 10^{-3}$. Using $V$ for phase space volume we have by definition $V_{j}=e^{s_{j} N}$, so obtaining the desired accuracy in $s_{j}$ to order $10^{-3}$ for each case requires a bound on the relative error for the volumes of $\Delta V_{j} / V_{j}<e^{N / 10^{3}}-1$.

A different way to make sense of perturbations of the fcc and hcp structures is to consider deviations of these structures that are periodic with some fixed period; if the density is high enough the two sets of configurations are disconnected and thus the volumes $V_{f c c}$ and $V_{h c p}$ are well defined. Both molecular dynamics [W1,W2] and Monte Carlo simulations [BFMH,BWA,MH] used this setup, although mostly at densities near melting, or equivalently, for spheres of radius about 0.9, where the two sets of configurations are actually connected to one another (for periods 6 or larger in each direction). At these densities, a simulation should theoretically sweep the entire phase space, regardless of where it is started. However, on the time scale available in practice the process is found to be trapped in regions whose configurations can be associated with a single lattice structure, and it is the volumes of these regions that have been used to conclude that fcc is the more stable structure. Although the (extremely large) fcc-hcp mixing time may not be a problem in these simulations, the success of the method does depend on the absence of relevant time scales beyond the ones that have been observed so far. Moreover, such simulations do not give any intuition into the mechanism, almost certainly geometric, which distinguishes these close packed structures.

We now consider the method of Stillinger et al, which does not (inherently) rely on simulation and does in principle allow for a geometric interpretation. The first nontrivial term in the expansion below has been analyzed in [RS]. Consider again our finite block of spheres frozen in a perfect crystal arrangement, fcc or hcp, at density below close packing, and a subset $Q$ of them which we free up. The entropy $S_{Q}$ of the moveable spheres associated with (or labelled by) the sites $Q$ can be written as an exact sum

$$
S_{Q}=\sum_{P \subseteq Q} C_{P}=\sum_{P \subset Q:|P|=1} C_{P}+\sum_{P \subset Q:|P|=2} C_{P}+\cdots
$$


which defines the correction terms $C_{P}$. For each $n>0$ there are, up to symmetries, a fixed number of contributing "polymers" $P$ of $|P|=n$ spheres; one can compute these and then each " $n$-body correction" $s_{n, Q}=\frac{1}{|Q|} \sum_{P \subseteq Q:|P|=n} C_{P}$, so that

$$
s_{Q}=\frac{S_{Q}}{|Q|}=\sum_{n \geq 1} s_{n, Q} .
$$

As $|Q| \rightarrow \infty$ the number of occurences per site of the polymer $P$ approaches a fixed frequency $f_{P}$, and $s_{n, Q}$ approaches a limit $s_{n}=\sum_{|P|=n} f_{P} C_{P}$. The entropy per sphere of the hard sphere solid is then $s=\sum_{n>1} s_{n}$.

We specialize to densities very close to the maximum density, that is, phase space volumes (and hence entropies) are computed only to leading order in the difference $\epsilon$ between the lattice spacing 1 and the sphere diameter $1-\epsilon$. As noted above $\exp \left(S_{P}\right)$ is the volume of a polyhedron in $3|P|$ dimensions, and the dimensions of this polyhedron all scale as $\epsilon$. In particular for $|P|=1, s_{1}=C_{P}$ is simply the logarithm of the scaled volume of a Voronoi cell for a sphere, and this value is the same for fcc as for hcp. Note that for $|P|>1$ the (divergent) $\ln (\epsilon)$ terms in $C_{P}$ cancel, so $s_{n}$ is well-defined in the $\epsilon \rightarrow 0$ limit for $n>1$.

It is natural to simply truncate the sum $\sum_{n} s_{n}$ and compute each $s_{n}$ by computing the appropriate volumes. See Table 1, below. The success of this method depends on the rapid decay of the individual terms $s_{n}$ with increasing $n$, so that the sum of uncomputed terms are convincingly negligible. Note that for both fcc and hcp, and for all computed levels, $s_{n+1}$ is in absolute value considerably smaller than $s_{n}$. Recalling that we want to estimate $\Delta s$ to within an error of $10^{-3}$, and assuming the two series remain geometrically decreasing by a factor of roughly 3, one needs to compute all terms up to level $n=7$. Unfortunately this seems unattainable with current computers as we discuss in the next section.

\begin{tabular}{|c|r|r|r|r|r|}
\hline$N$ & \multicolumn{1}{|c|}{ fcc: $s_{n}$} & fcc: $\sum_{1}^{n} s_{m}$ & hcp: $s_{n}$ & hcp: $\sum_{1}^{n} s_{m}$ & \multicolumn{1}{c|}{$\Delta s$} \\
\hline 1 & 1.0986122 & 1.0986122 & 1.0986122 & 1.0986122 & 0.0000000 \\
\hline 2 & -0.1647410 & 0.9338712 & -0.1636474 & 0.9349648 & -0.0010935 \\
\hline 3 & -0.0517903 & 0.8820808 & -0.0518249 & 0.8831398 & -0.0010589 \\
\hline 4 & -0.0274325 & 0.8546482 & -0.0261820 & 0.8569577 & -0.0023094 \\
\hline 5 & 0.0060605 & 0.8607088 & 0.0026023 & 0.8595601 & 0.0011486 \\
\hline
\end{tabular}

Table 1. Partial entropies for fcc and hcp structures.

In addition to computing and adding terms in order, we can examine the contributions of individual polymers. Figure 1, below, shows the distribution of entropy corrections $C_{p}$ that contribute to $s_{5}$, after convolution by a Gaussian with variance $10^{-10}$. Note that the contribution of individual polymers should not be viewed as independent random variables. The sum of the $\sim 500$ terms contributing to $s_{5}$ for 
hcp is not of order $\sqrt{500}$ larger than a typical term: rather, it is the same size as a typical term. Likewise for $s_{3}, s_{4}$, and for fcc. Hopefully, by studying the distribution of terms contributing to $s_{3}, s_{4}$ and $s_{5}$ we will be able to devise resummation schemes with accelerated convergence.

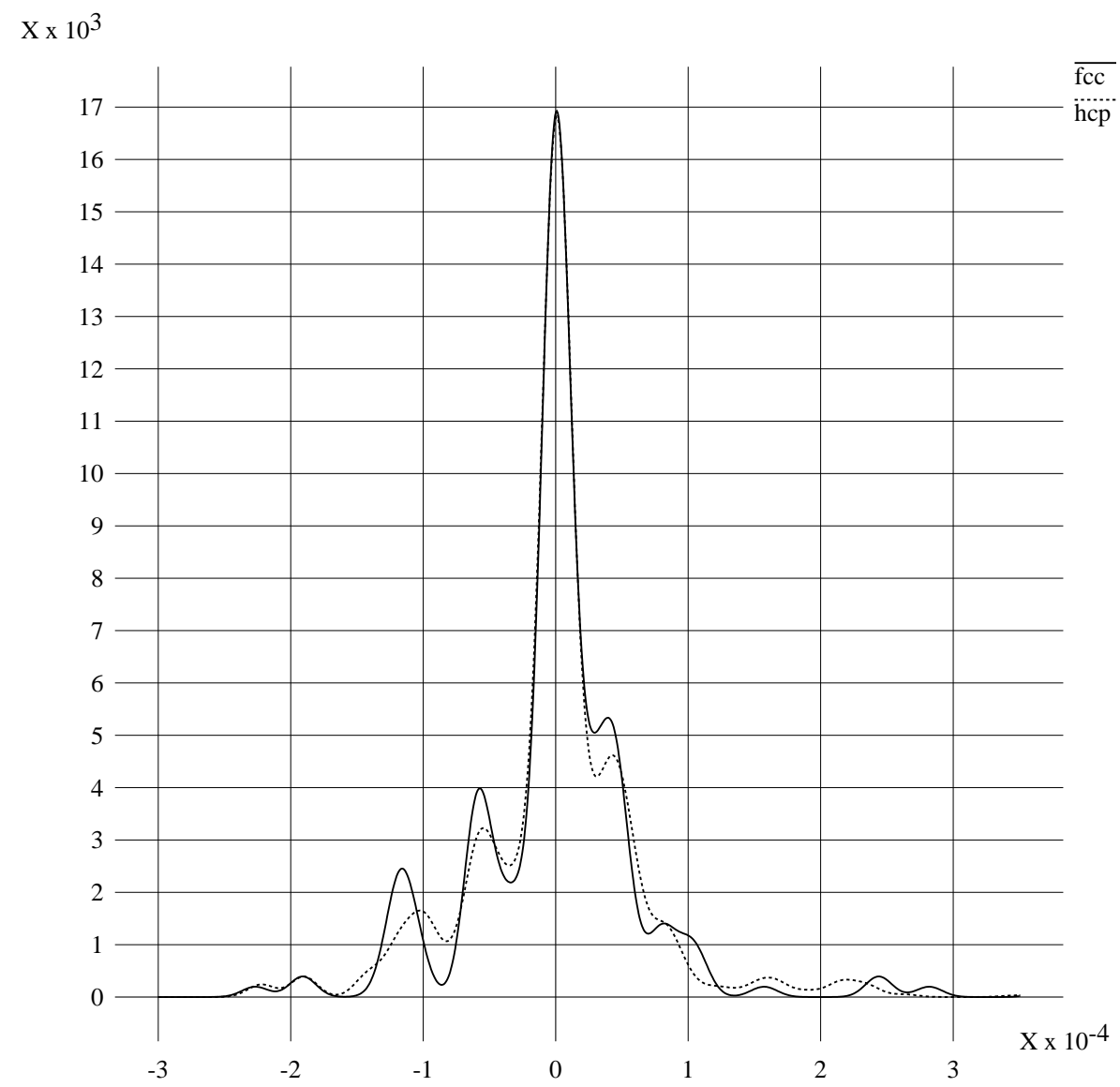

Figure 1. Distribution of entropy corrections $C_{P}$ contributing to $s_{5}$.

Another important goal is to understand the geometric mechanisms underlying the difference in entropy between fcc and hcp. The qualitative differences between the terms contributing to $s_{2}$ were considered in [RS]. Although some general patterns have emerged from the data presented here (e.g., compact polymers tend to contribute negatively, while extended polymers tend to contribute positively), a true geometric understanding still eludes us.

\section{Computation}

The computations by Stillinger et al of ( $s_{2}$ and) $s_{3}$ to 5 -digit accuracy was remarkable for the time. We have taken advantage of current computers to compute $s_{n}$ up to $n=5$. To obtain $s_{5}$ we needed to compute among other things the volumes of 591 polyhedra in 15 dimensions (the phase space for 5 spheres). The basic algorithm is simple, based on successive "triangularizations": starting with $d=15$, 
choose a point in the $d$-dimensional polyhedron to serve as the common apex of pyramids with the faces of the polyhedron as bases, and then add up the pyramid volumes $A B / d$, where $A$ is the altitude and $B$ the volume of the base. Given that the base is again a polyhedron, now in dimension $d-1$, the process can be iterated. The main drawback of this algorithm is that the same lower dimensional volumes are computed repeatedly in different branches of the recursion. In the case of our 15-dimensional polyhedra, which have on average 55 faces, this leads to a highly impractical number of computations. The standard way of dealing with this problem is to store and to re-use the results from previous computations. Programs that implement this strategy are readily available, but for reasons of efficiency (the program that was recommended to us was too slow by several orders of magnitude) we wrote the necessary code ourselves; it is available at xx. Volumes in 15 dimensions each took between 7 and 160 hours of computation time, with an average of 29 hours, on a 64-bit processor running at $2.2 \mathrm{GHZ}$, using $2 \mathrm{~GB}$ of memory. The number of re-used lower dimensional volumes in each of these cases is of the order of $10^{10}$. Graphs of the entropy corrections $f_{P} C_{P}$ associated with the volumes $P$ are shown in Fig 1 .

We do not expect such an exact computation of $s_{6}$ to be practical before substantial progress in computer hardware.

\section{References}

[B] J. A. Barker, Lattice Theories of the Liquid State (Macmillan, New York, 1963).

[BWA] A. D. Bruce, N. B. Wilding, and G. J. Ackland, Phys. Rev. Lett. 79, 3002 (1997).

[BFMH] P. G. Bolhuis, D. Frenkel, S.-C. Muse, and D. A. Huse, Nature (London) 388, 235 (1997).

[FL] D. Frenkel and A. Ladd, J. Chem. Phys. 81, 3188 (1984)

[HR] W. Hoover and F. Ree, J. Chem. Phys. 49, 3609 (1968).

[MH] S.-C. Mau and D. A. Huse, Phys. Rev. E 59, 4396 (1999).

[RS] C. Radin, and L. Sadun, Phys. Rev. Lett. 94, 015502 (2005).

[RSYS] W. G. Rudd, Z.W. Salsburg, A. P. Yu, and F. H. Stillinger, J. Chem. Phys. 49, 4857 (1968).

[W1] L.V. Woodcock, Nature (London) 385, 141 (1997).

[W2] L.V. Woodcock, Nature (London) 388, 236 (1997). 